\title{
In-situ X-ray Observation of Synthesizing Process for Rare-earth Transition-metal Pnictides under High Temperature and High Pressure
}

\author{
Chihiro Sekine, ${ }^{1 *}$ Hidetoshi Osanai, ${ }^{1}$ Keisuke Ikemori, ${ }^{1}$ Ryosuke Nakajima, ${ }^{1}$ Shingo Deminami, Jirattagan Sirimart ${ }^{1}$ and Hirotada
} Gotou $^{2}$

We have performed in-situ x-ray diffraction (XRD) measurements in order to acquire the information about synthesis conditions of several rare-earth transition-metal pnictides. By considering the results for a battery of the in-situ XRD measurements, we have obtained conditions optimal for synthesizing layered rare-earth zinc phosphides $R \mathrm{Zn}_{3} \mathrm{P}_{3}(R=\mathrm{Y}$ and Dy) and a new filled skutterudite compound $\mathrm{Tb}_{x} \mathrm{Co}_{4} \mathrm{Sb}_{12}$. According to the optimum condition acquired by the in-situ XRD experiments, we have succeeded in synthesizing high-quality samples.

Keywords: High-temperature and high-pressure synthesis; Rare-earth transition-metal pnictides; In-situ x-ray diffraction

Received 13 August 2018, Accepted 24 September 2018

DOI: $10.30919 / \mathrm{esmm} 5 \mathrm{f} 105$

\section{Introduction}

Transition metal pnictides have attracted much attention for their various anomalous physical properties and application as functional materials such as thermoelectric (TE) materials, superconductors and catalysts. ${ }^{1-7}$ However, a synthesis of good quality samples free from secondary phases or impurities for transition-metal pnictides is extremely difficult at ambient pressure because the vapor pressure of pnictogen is considerably high. The high-temperature and highpressure (HTHP) synthesis is one of the most effective methods to prepare transition-metal pnictides. ${ }^{8}$ Nevertheless, HTHP synthesis practically takes long time and much effort to decide the optimum conditions to synthesize high-quality samples or new compounds. Therefore, it is extremely efficient to search the processes for synthesis of target materials at HTHP by in-situ x-ray diffraction (XRD) measurements in advance. ${ }^{8-10}$

In this study, we focus on two systems of rare-earth transitionmetal pnictides because both systems are important for future application as functional materials. First system is skutterudite compounds, which are expected as high-performance TE materials, and the other system is layered rare-earth zinc phosphides $R \mathrm{Zn}_{3} \mathrm{P}_{3}(R$ $=$ rare-earth element), which are magnetic frustrated materials and have a possibility of multiferroic. However, a synthesis of good quality samples for both systems is extremely difficult at ambient pressure. Therefore, we have performed in-situ XRD measurements

${ }^{I}$ Graduate School of Engineering, Muroran Institute of Technology, Muroran, Hokkaido 050-8585, Japan

${ }^{2}$ Institute for Solid State Physics, University of Tokyo, Kashiwa, Chiba 277-8581, Japan

*E-mail: sekine@mmm.muroran-it.ac.jp for the systems in order to synthesize good quality samples free from secondary phases or impurities under high pressure.

The skutterudite family includes binary, unfilled and ternary, filled types with the same space group. The binary compounds have the general expression $M X_{3}$ or $\square M_{4} X_{12}$, where $M$ is transition metal such as Co, Rh and Ir (site $8 \mathrm{c}$ ), $X$ is pnictogen (site $24 \mathrm{~g}$ ), and the symbol $\square$ stands for a vacancy (site $2 \mathrm{a}$ ) in the large cages formed by the $\mathrm{M}$ and $\mathrm{X}$ ions. The crystal structure of unfilled skutterudite compounds is body centered cubic ( $\mathrm{CoAs}_{3}$ type), space group is $\operatorname{Im} \overline{3}$ $\left(T_{\mathrm{h}}^{6}\right.$, No. 204). ${ }^{11}$ This system is most promising high-performance TE materials. The TE performance is estimated by the figure of merit $Z$ $=\sigma S^{2} / \kappa$. Here, $\sigma, \kappa$ and $S$ are the electrical conductivity, the thermal conductivity and the Seebeck coefficient, respectively. Furthermore, $\kappa$ is given by $\kappa_{\mathrm{E}}+\kappa_{\mathrm{L}}$, where $\kappa_{\mathrm{E}}$ and $\kappa_{\mathrm{L}}$ are electronic and lattice contribution, respectively. ${ }^{12}$ The binary compound $\mathrm{CoSb}_{3}$ (or $\square \mathrm{Co}_{4} \mathrm{Sb}_{12}$ ) exhibits superior TE properties (large $S$ and high hole mobility). ${ }^{13}$ In contrast to the large power factor $\sigma S^{2}$ of $\mathrm{CoSb}_{3}, \kappa_{\mathrm{L}}$ is high. However, the weak point can be improved by partially filling guest ions into the vacancy $\square$ of $\square \mathrm{Co}_{4} \mathrm{Sb}_{12}$. It is conceived that the guest ions located inside the vacancy exhibit a random motion (rattling) around the equilibrium positions. ${ }^{2}$ Then, the rattling causes a significant reduction of $\kappa_{\mathrm{L}}$ by a marked phonon scattering. Actually, it was reported that the vacancy of $\mathrm{CoSb}_{3}$ could be partially filled by rare-earth $(R)$ ions and a significant reduction of $\kappa_{\mathrm{L}}$ were observed for $R_{x} \mathrm{Co}_{4} \mathrm{Sb}_{12}{ }^{13,14}$ In order to cause a significant rattling effect, smaller and heavier ions such as heavy rare-earth ions could be more efficient. Therefore, heavier $R$ ions could be desirable for guest ions filled the vacancy of $\mathrm{CoSb}_{3}$. However, synthesis of heavy rare earth (except for $\mathrm{Yb}$ ) filled $\mathrm{CoSb}_{3}$ has not been reported so far while $R$ ions such as $\mathrm{La}, \mathrm{Ce}, \mathrm{Nd}$ and $\mathrm{Eu}$ have been successfully filled into the voids of $\mathrm{CoSb}_{3}$ by means of conventional methods at ambient 
pressure. $^{13-16}$ Mei et al. have carried out a systematic theoretical research of the filling fraction limit (FFL) of $R$ atoms in $\mathrm{CoSb}_{3}$ at ambient pressure. ${ }^{17}$ Based on their calculations, the FFL decreases rapidly from $\mathrm{La}$ to $\mathrm{Sm}$, and becomes zero for heavy rare earths from $\mathrm{Gd}$ to $\mathrm{Lu}$, with the exception of Eu and $\mathrm{Yb}$. However, high pressure could benefit the entrance of heavier rare earths into the vacancy of $\mathrm{CoSb}_{3}$ than ambient pressure. Therefore, we have currently tried to synthesize partially heavy rare earth (Gd-Lu) filled $\mathrm{CoSb}_{3}$, systematically. Among them, we introduce the results of partially $\mathrm{Tb}$ (which is one of heavy rare earth elements) filled $\mathrm{CoSb}_{3}\left(\mathrm{~Tb}_{x} \mathrm{Co}_{4} \mathrm{Sb}_{12}\right)$ by the HTHP synthesis as an example. As far our knowledge, $\mathrm{Tb}_{x} \mathrm{Co}_{4} \mathrm{Sb}_{12}$ is a new compound.

Then, we focus on layered rare-earth zinc phosphides $R \mathrm{Zn}_{3} \mathrm{P}_{3}(R$ $=$ rare-earth element). The crystal structure of layered rare-earth zinc phosphides $R \mathrm{Zn}_{3} \mathrm{P}_{3}\left(R=\right.$ rare-earth element) is hexagonal $\left(\mathrm{ScAl}_{3} \mathrm{C}_{3}\right.$ type), space group is $P 6_{3} / m m c\left(D_{6 h}^{4}\right.$, No. 194) ${ }^{18}$ The $\mathrm{Zn}$ and $\mathrm{P}$ atom form hexagonal net, wherein the $\mathrm{Zn}$ and $\mathrm{P}$ atoms alternate. The $R$ atoms form layers separating the $\mathrm{Zn}-\mathrm{P}$ nets and a two-dimensional triangular lattice. Therefore, the compounds are expected as a frustrated system. Since the discovery of the giant magnetoelectric effects in a perovskite manganite $\mathrm{TbMnO}_{3},{ }^{19}$ multiferroics studies have become active. Multiferroics have potential for applications as next generation devices such as magnetic field sensors, actuators, switching device and new types of memory. ${ }^{20}$ Many maltiferroics have been discovered in magnetic materials with a geometrical frustration in the crystal structure. ${ }^{19,21,22}$ There is a possibility that $R \mathrm{Zn}_{3} \mathrm{P}_{3}(R=$ rare-earth element $)$ is also a magnetic frustrated system. However, the physical properties for most of $R \mathrm{Zn}_{3} \mathrm{P}_{3}$ except $C e \mathrm{Zn}_{3} \mathrm{P}_{3}$ have not been studied so far, while the synthesis of the phosphides by a flux method was reported. ${ }^{18}$ It was reported that $\mathrm{CeZn}_{3} \mathrm{P}_{3}$ exhibits an antiferromagnetic ordering at $T_{\mathrm{N}}=0.8 \mathrm{~K}$. The entropy of the magnetic contribution is $\sim 0.4 R \ln 2$ at $T_{\mathrm{N}}$, and reaches $R \ln 2$ around $10 \mathrm{~K}^{23}$ This suggests that $\mathrm{CeZn}_{3} \mathrm{P}_{3}$ has the short-range magnetic interaction above $T_{\mathrm{N}}$ and the compound could be a frustrated system. In order to study $R Z_{3} \mathrm{P}_{3}$ systematically, we have tried to synthesize DyZn $\mathrm{P}_{3}$ with a large magnetic moment of Dy and $\mathrm{YZn}_{3} \mathrm{P}_{3}$ as a nonmagnetic reference under high pressure at the beginning. The high-pressure synthesis of $R \mathrm{Zn}_{3} \mathrm{P}_{3}(R=$ rare-earth element) has not been reported so far. Therefore, we have performed in-situ XRD measurements to search the processes for synthesis of $\mathrm{DyZn}_{3} \mathrm{P}_{3}$ and $\mathrm{YZn}_{3} \mathrm{P}_{3}$ at HTHP.

As a pressurization method for high-pressure experiments using quantum beams such as synchrotron X-ray, neutron, and muon, multi-anvil assembly 6-6 (MA6-6) with a DIA-type cubic-anvil high-pressure apparatus is becoming to be a standard. ${ }^{24}$ MA6-6 consists of six small second-stage anvils with an anvil guide. Recently, we have developed a new anvil guide, which can make it much easier to perform the experiments using MA6-6. In this paper, we also present a detailed design of the new anvil guide for MA6-6 and report results of pressure generation tests.

\section{Experimental}

In-situ XRD measurements at HTHP have been performed at Photon Factory (PF) in High Energy Accelerator Research Organization (KEK) (Tsukuba, Japan). XRD patterns were taken by an energydispersive method using synchrotron radiation (white X-ray) and a solid-state detector at the beam line AR-NE5C. ${ }^{8-10}$ High pressure was applied using multi-anvil assembly 6-6 (MA6-6) with a DIA-type cubic-anvil high-pressure apparatus, the MAX80 system, installed at the beam line. MA6-6 consists of six small second-stage anvils with an anvil guide and can be compressed by a DIA-type cubic-anvil apparatus. ${ }^{24}$ We have to set the second stage anvils at the accurate positions of the DIA geometry, carefully. Therefore, an anvil guide is really needed. The anvil guide (frame) is generally made of tool steel (SUS304) and the holes are opened at the frame along one of the diagonal direction for access to the incident and diffracted X-rays. ${ }^{8}$ Fig. 1a shows a schematic illustration of MA6-6



(a)

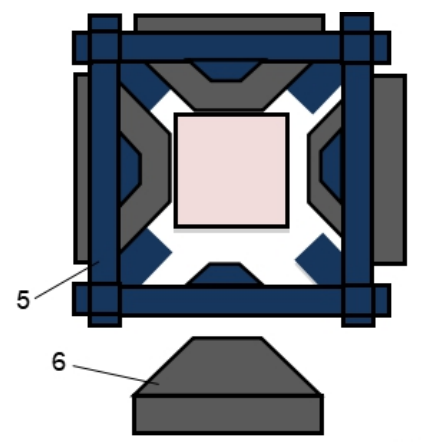

(b)
Fig. 1 Schematic illustration of MA6-6 using a regular steel frame (a) and MA6-6 using a plastic frame (b) 1, sample cell; 2, anvil guide (steel frame); 3, spacer glued to the anvil guide (balsa); 4, spacer glued to the anvil (Teflon); 5, anvil guide (plastic frame); 6, second stage anvil.

using a conventional steel frame. The spacers (Teflon) are necessary in setting the second stage anvils at their initial positions in the DIA geometry. Furthermore, we glue four spacers (balsa) on each outer surface of the anvil guide. When pressurizing is carried out, these spacers make it possible that second stage anvils contact with the first stage anvils tenderly because the first stage anvils compress the spacers first and then contact the second stage anvils.

Although the use of MA6-6 simplifies the anvil replacement process, we have to still consume time and labor for setting, namely, we have to attach an electric insulating tapes on the surfaces of the steel frame and build up many parts (spacers). This process takes long time (several hours) because the anvil positions should be adjusted carefully. Therefor, we developed an integrated-type plastic frame (Fig. 1b) using 3D printer (KEYENCE, AGILISTA-3200). Fig. 2a shows a design of a plastic anvil guide integrated with spacers, which are also made of the same plastic. It is our original idea that plastic spacers are integrally molded on the plastic anvil guide using $3 \mathrm{D}$ printer. Fig. $2 \mathrm{~b}$ and $\mathrm{c}$ show photographs of the plastic frame made by $3 \mathrm{D}$ printer and MA6-6 with the plastic frame set on the first stage anvils of a DIA-type cubic-anvil high-pressure apparatus, the MAX80 system, respectively. The plastic anvil guide with spacers can make it much easier to assemble MA6-6 because we do not have to attach electric insulating tapes on the surfaces of the anvil guide and do not have to glue spacers on the anvils and the anvil guide. We can just insert the anvils to the frame and the anvils locate at accurate positions automatically.

Pressure generation of the MA6-6 with the plastic frame was tested using the MAX80 system installed at PF, KEK. Pressure was determined by the lattice constant of the $\mathrm{NaCl}$. Pyrophyllite cube with edge length of $7 \mathrm{~mm}$ was used as the pressure medium. The truncated edge length (TEL) of the second-stage anvil made of tungsten carbide (WC) is $4 \mathrm{~mm}$. The anvil guide made of plastic with an outer edge length of $28 \mathrm{~mm}$ was used. The anvil guide made of tool steel (SUS304) was also used for comparison. The TEL of the first-stage anvil is $27 \mathrm{~mm}$. Fig. 3 shows the determined pressure calibration curves. The plastic frame can be applied without 


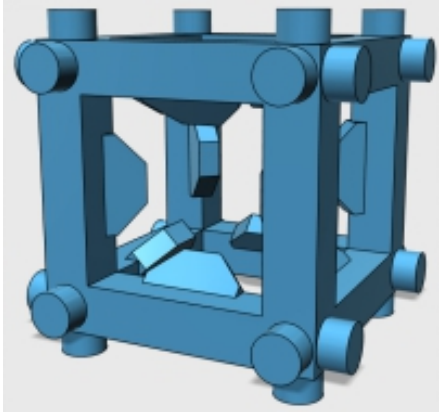

( a )

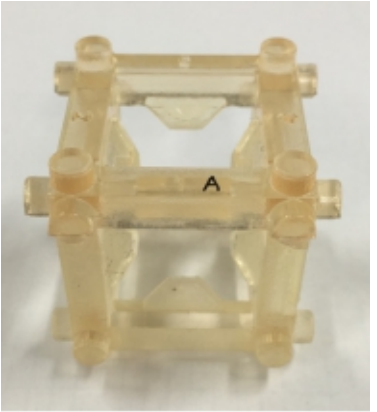

( b )

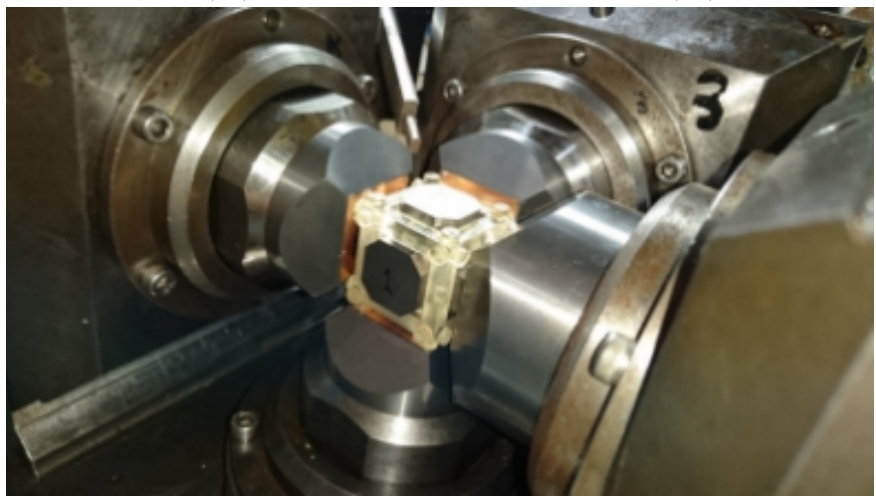

( c )

Fig. 2 Design of a plastic frame integrated with spacers (a) a photo of the plastic frame made by 3D printer (b), and a photo of MA6-6 using the plastic frame set on the first stage .



Fig. 3 Generated pressure plotted as a function of applied load for a combination of MA6-6 with the plastic frame and DIA-type cubicanvil high-pressure apparatus. The TEL of the second stage anvil is 4 $\mathrm{mm}$. The anvil guide made of tool steel (SUS304) was also used for comparison.

problems while the curve for the plastic frame is slightly less efficient than that for a regular steel frame.

The sample cell assembly of the in-situ XRD measurements for rare-earth transition-metal pnictides is almost the same as that in previous report. ${ }^{8}$ The starting materials, which are mixture of each element chips or powders, are put in a boron nitride $(\mathrm{BN})$ crucible. The crucible with a graphite electric heater is inserted in a cubic solid pressure medium made of pyrophyllite. Pressure is determined by the lattice constant of the $\mathrm{NaCl}$ internal pressure marker.

In order to prepare large bulk samples at HTHP, a double-stage multi-anvil high-pressure apparatus (Kawai type system) was used (quench experiments). ${ }^{8}$ Pressurizing method of the Kawai-type system is as follows. First, a hydraulic ram drives six first-stage anvils. Then, the six first-stage ones compress eight second-stage cubic anvils. Finally, the second-stage ones compress an octahedral solid pressure medium made of magnesia $\left(\mathrm{MgO}+5 \% \mathrm{Cr}_{2} \mathrm{O}_{3}\right)$. For the second-stage anvils, WC cubes with a TEL of $11 \mathrm{~mm}$ were used. The sample cell assembly is similar to that used for in-situ experiments. The pressure medium was transformed into an octahedron with a length of each side of $18 \mathrm{~mm}$. The starting substances, which are mixture of each element chips or powders, were put in a crucible made of BN. The crucible, with a graphite heater surrounded with a zirconia $\left(\mathrm{ZrO}_{2}\right)$ thermal insulator, was inserted into the magnesia octahedron. The prepared samples were characterized by powder x-ray diffraction using Co $K \alpha_{1}$ radiation and silicon as a standard.

\section{Results and discussion}

In the following sections, results of the in-situ XRD of synthesizing process under HTHP for skutterudites and layered rare-earth phosphides $R \mathrm{Zn}_{3} \mathrm{P}_{3}$ will be reported.

\subsection{Skutterudite}

We have performed in-situ XRD measurements of synthesizing process for $\mathrm{CoSb}_{3}$ as a test run of MA6-6 with the plastic frame. Fig. 4 shows XRD patterns of synthesizing process of $\mathrm{CoSb}_{3}$ at $2 \mathrm{GPa}$. Fig. 4a depicts XRD pattern of the starting materials, which are mixture of $\mathrm{Co}$ and $\mathrm{Sb}$ powders in the atomic ratio of $\mathrm{Co}: \mathrm{Sb}=1: 3$, at room temperature (RT) and ambient pressure. Solid triangles and solid circles indicate the Bragg peaks of $\mathrm{Co}$ and $\mathrm{Sb}$, respectively. Solid squares designate the characteristic $\mathrm{x}$-ray for $\mathrm{Sb}$. Fig. $4 \mathrm{~b}$ shows XRD pattern at RT after applying pressure up to $2 \mathrm{GPa}$. The peak positions of the starting materials shifted with increasing pressure and any additional diffraction with pressure was not observed. Accompanying the temperature rise, the Bragg peaks of the starting materials faded out, and then the peaks of the skutterudite structure was observed above $500{ }^{\circ} \mathrm{C}$. Fig. $4 \mathrm{c}$ shows XRD pattern at $2 \mathrm{GPa}$ and $530{ }^{\circ} \mathrm{C}$. The diffraction peaks of $\mathrm{CoSb}_{3}$ were observed in addition to those of the starting materials. The numbers indicate the plane index of skutterudite structure. All diffraction lines were indexable using the skutterudite structure at $630{ }^{\circ} \mathrm{C}$ (Fig. $\left.4 \mathrm{~d}\right)$. Then, the peaks of a secondary phase $\left(\mathrm{CoSb}_{2}\right)$, which are shown by crosses, appeared at $915^{\circ} \mathrm{C}$ (Fig. 4e). Further, most of the diffraction peaks disappeared and only the peaks of the characteristic $\mathrm{x}$-ray of Sb were observed at $1050{ }^{\circ} \mathrm{C}$ (Fig. 4f). This indicates that the sample melts at this temperature. By considering the results, we obtained an appropriate temperature range of $650-850{ }^{\circ} \mathrm{C}$ for synthesizing $\mathrm{CoSb}_{3}$ at $2 \mathrm{GPa}$. This result is consistent with the previous report ${ }^{9}$. Moreover, it could be confirmed that the MA6-6 system with the plastic frame is available for experiments at temperature up to 1050 ${ }^{\circ} \mathrm{C}$.

Then, we have performed in-situ XRD experiments of synthesizing process for partially filled skutterudite compound 

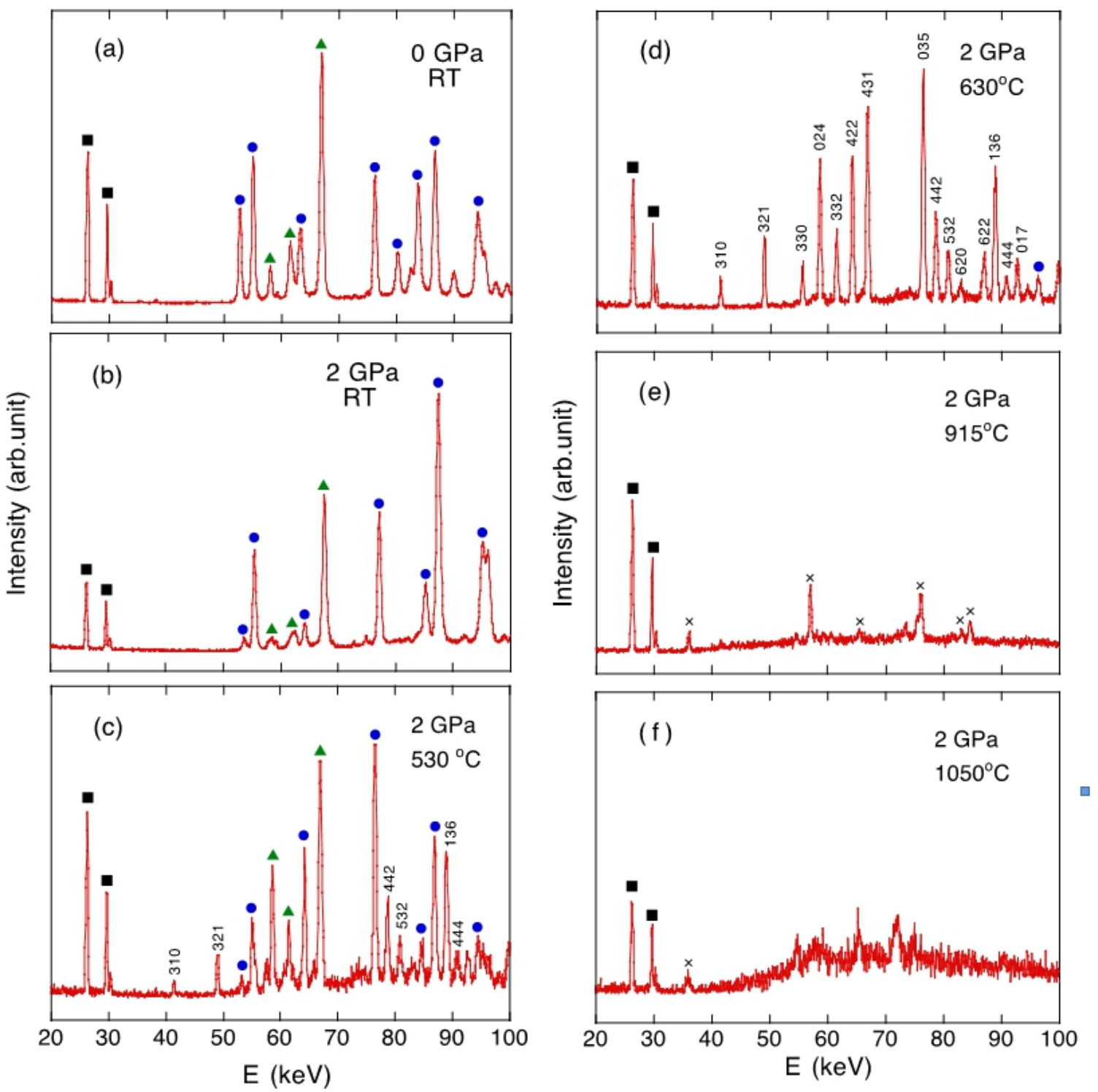

Fig. $4 \mathrm{XRD}$ patterns of synthesizing process of $\mathrm{CoSb}_{3}$ at $2 \mathrm{GPa}$. Solid triangles and circles indicate the Bragg peaks of Co and $\mathrm{Sb}$, respectively. Solid squares designate the characteristic x-ray of Sb. Numbers in the spectra are the Miller index of the Bragg peaks for CoSb ${ }_{3}$. Crosses show the peaks of a secondary phase. The starting materials at room temperature (RT) and ambient pressure (a), RT and $2 \mathrm{GPa}(\mathrm{b}), 530{ }^{\circ} \mathrm{C}(\mathrm{c}), 630{ }^{\circ} \mathrm{C}$ (d), $915^{\circ} \mathrm{C}(\mathrm{e})$ and $1050^{\circ} \mathrm{C}(\mathrm{f})$.

$\mathrm{Tb}_{x} \mathrm{Co}_{4} \mathrm{Sb}_{12}$ at $4 \mathrm{GPa}$. Starting substances are mixture of Tb chip, Co and $\mathrm{Sb}$ powders in the atomic ratio of $\mathrm{Tb}: \mathrm{Co}: \mathrm{Sb}=0.5: 4: 12$. By considering the results for a battery of the in-situ XRD measurements, we obtained an appropriate temperature range of 500-700 ${ }^{\circ} \mathrm{C}$ for synthesizing $\mathrm{Tb}_{x} \mathrm{Co}_{4} \mathrm{Sb}_{12}$ at $4 \mathrm{GPa}$. Then, we have actually synthesized $\mathrm{Tb}_{x} \mathrm{Co}_{4} \mathrm{Sb}_{12}$ at $4 \mathrm{GPa}$ and $600{ }^{\circ} \mathrm{C}$ using a Kawaitype multi-anvil high-pressure apparatus according to the optimum condition acquired by the in-situ XRD experiments. Figure 5 shows the XRD pattern of $\mathrm{Tb}_{x} \mathrm{Co}_{4} \mathrm{Sb}_{12}$ prepared under high pressure using mixture of $\mathrm{Tb}$ chip, $\mathrm{Co}$ and $\mathrm{Sb}$ powder in the atomic ratio of $\mathrm{Tb}: \mathrm{Co}$ $: \mathrm{Sb}=0.5: 4: 12$ as the starting materials. The most of the observed diffraction lines were indexable using the skutterudite structure. The lattice constant determined by a least-squares fit to the data was $9.049 \AA$. The lattice constant of $\mathrm{Tb}_{x} \mathrm{Co}_{4} \mathrm{Sb}_{12}$ is larger than that of $\mathrm{CoSb}_{3}$. This suggests that the filling of $\mathrm{Tb}$ to $\mathrm{CoSb}_{3}$ was succeeded.

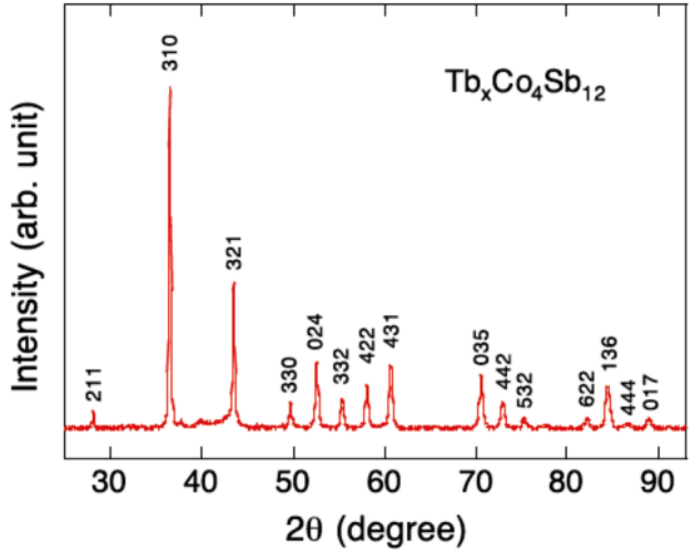

Fig. 5 XRD pattern of $\mathrm{Tb}_{x} \mathrm{Co}_{4} \mathrm{Sb}_{12}$ prepared under high pressure. 


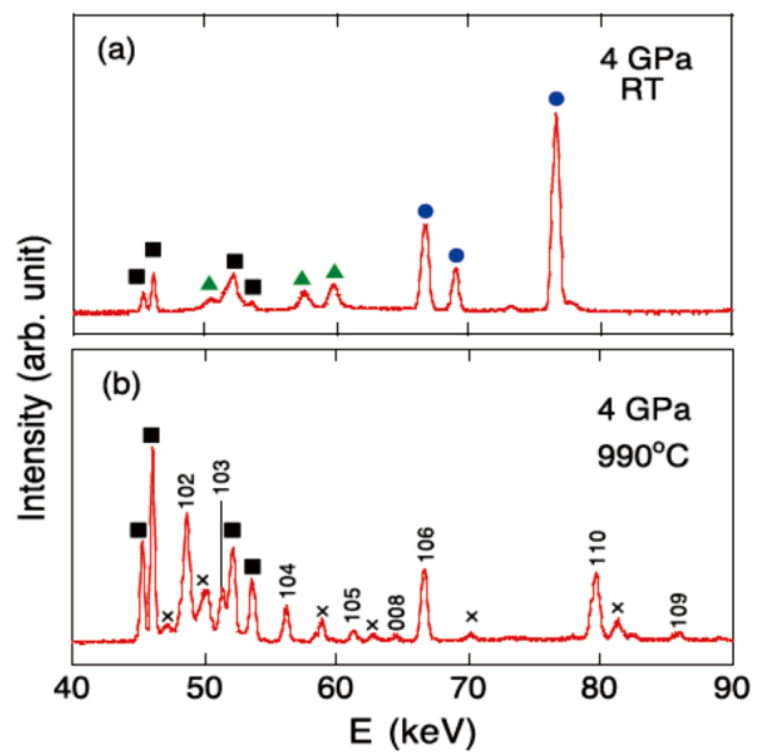

Fig. 6 XRD patterns of synthesizing process of DyZn ${ }_{3} \mathrm{P}_{3}$ at $4 \mathrm{GPa}$. Solid triangles and circles indicate the Bragg peaks of $\mathrm{Zn}$ and Dy, respectively. Solid squares designate the characteristic x-ray of Dy. Numbers in the spectra are the Miller index of the Bragg peaks for DyZn $\mathrm{n}_{3} \mathrm{P}_{3}$. Crosses indicate the peaks of secondary or impurity phases $\left(\mathrm{ZnP}_{2}, \mathrm{Z}_{3} \mathrm{P}_{2}\right.$ and DyZnPO). The starting substances at room temperature (RT) and $990{ }^{\circ} \mathrm{C}(\mathrm{b})$.

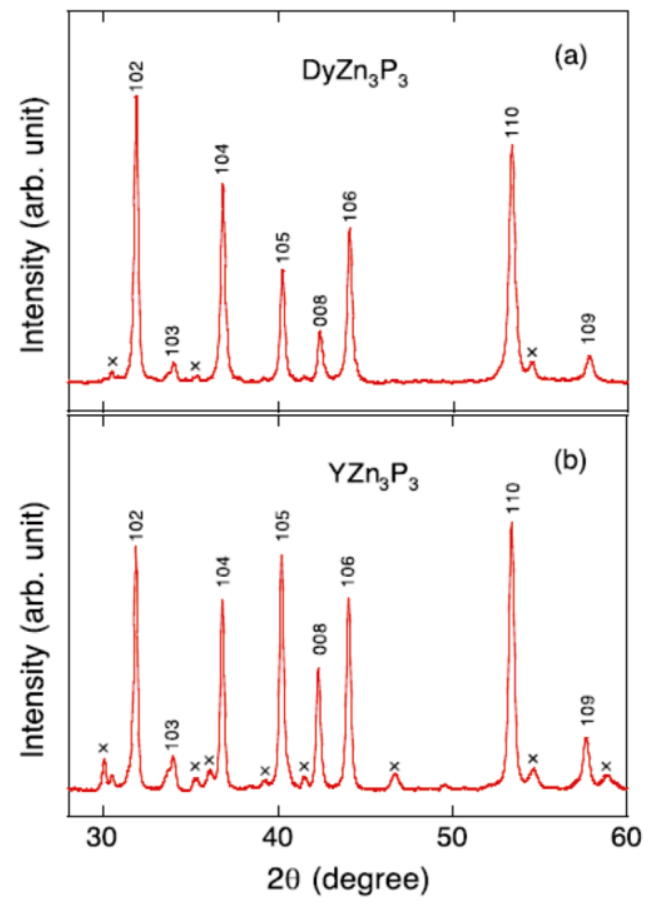

Fig. 7 XRD pattern of $\operatorname{DyZn}_{3} \mathrm{P}_{3}$ (a) and $\mathrm{Y} \mathrm{Zn}_{3} \mathrm{P}_{3}$ (b) synthesized under high pressure. Crosses indicate the peaks of secondary or impurity phases $\left(\mathrm{Zn}_{3} \mathrm{P}_{2}\right.$ and DyZnPO or YZnPO).
To determine the actual filling rate of $\mathrm{Tb}$, the chemical compositional analysis was conducted using a scanning electron microscope (SEM) with an energy dispersive X-ray spectrometer (EDX) (JEOL 6510). For the point analysis, 8 to 10 different points were carefully chosen to reduce the errors. The actual filling ratio estimated by SEM-EDX was found to be $x=0.11$. Although the theoretical calculations indicate that $\mathrm{Tb}$ with relatively smaller ionic radii cannot be inserted into the lattice voids of $\mathrm{CoSb}_{3}(\mathrm{FFL}=0)$ at ambient pressure, ${ }^{17}$ we have succeeded in synthesizing $10 \% \mathrm{~Tb}$ filled $\mathrm{Co}_{4} \mathrm{Sb}_{12}$ under high pressure. $\mathrm{Tb}_{x} \mathrm{Co}_{4} \mathrm{Sb}_{12}$ is a new compound.

\subsection{Layered rare-earth zinc phosphide}

We have also performed in-situ experiments for layered rare-earth zinc phosphide DyZn $\mathrm{P}_{3}$ at $4 \mathrm{GPa}$. Fig. 6a shows the XRD pattern of the starting substances, which are mixture of Dy chip, $\mathrm{Zn}$ and $\mathrm{P}$ powders in the atomic ratio of $\mathrm{Dy}: \mathrm{Co}: \mathrm{Sb}=1: 3: 3$, at room temperature (RT) and $4 \mathrm{GPa}$. Solid triangles and solid circles indicate the Bragg peaks of $\mathrm{Zn}$ and Dy, respectively. Solid squares designate the characteristic $\mathrm{x}$-ray of Dy. Accompanying the temperature rise, the Bragg peaks of the starting substances faded out, and then the peaks of the $\mathrm{ScAl}_{3} \mathrm{C}_{3}$ type structure were detected above $900{ }^{\circ} \mathrm{C}$. Fig. $6 \mathrm{~b}$ shows the XRD pattern at $4 \mathrm{GPa}$ and $990{ }^{\circ} \mathrm{C}$. Most diffraction lines were indexable using the $\mathrm{ScAl}_{3} \mathrm{C}_{3}$ type structure while the peaks of secondary phases $\left(\mathrm{ZnP}_{2}, \mathrm{Z}_{3} \mathrm{P}_{2}\right.$ and DyZnPO), which are indicated by crosses, were observed. We also performed the similar experiments for $\mathrm{YZn}_{3} \mathrm{P}_{3}$. By considering the results for a battery of the in-situ XRD measurements, we obtained an appropriate temperature around 980 amd $950{ }^{\circ} \mathrm{C}$ for synthesizing $\mathrm{DyZn}_{3} \mathrm{P}_{3}$ and $\mathrm{YZn}_{3} \mathrm{P}_{3}$ at $4 \mathrm{GPa}$. Based on the results of in-situ experiments, we have succeeded in synthesizing large bulk samples of $\mathrm{YZn}_{3} \mathrm{P}_{3}$ and $\mathrm{DyZn}_{3} \mathrm{P}_{3}$ under high pressure (quench experiment). Fig. 7 shows the powder XRD patterns of $\mathrm{DyZn}_{3} \mathrm{P}_{3}$ and $\mathrm{YZn}_{3} \mathrm{P}_{3}$ synthesized under high pressure. Although a small quantity of impurity phases $\left(\mathrm{Zn}_{3} \mathrm{P}_{2}\right.$ and DyZnPO or $\left.\mathrm{YZnPO}\right)$ was detected, most of the observed diffraction peaks were using the $\mathrm{ScAl}_{3} \mathrm{C}_{3}$ type structure.

The lattice constants of the phosphides determined by a leastsquare fit to the data are summarized in Table 1 . The obtained lattice constants are consistent with reported value. ${ }^{18}$

Tables 1 Crystal data and lattice parameters decided by XRD of layered rare-earth zinc phosphides $R \mathrm{Zn}_{3} \mathrm{P}_{3}(R=\mathrm{Y}$ and Dy) prepared under high pressure.

\begin{tabular}{|c|c|c|c|c|c|c|}
\hline \multirow{3}{*}{ Compound } & \multirow{3}{*}{ Crystal system } & \multirow{3}{*}{ Space group } & \multicolumn{4}{|c|}{ Lattice parameter } \\
\hline & & & \multicolumn{2}{|c|}{ This work } & \multicolumn{2}{|c|}{ Reference 18} \\
\hline & & & $\mathrm{a}(\AA)$ & c $(\AA)$ & $\mathrm{a}(\AA)$ & $\mathrm{c}(\AA)$ \\
\hline $\mathrm{YZn}_{3} \mathrm{P}_{3}$ & Hexagonal & $\mathrm{Pb}_{3} / m m c$ & 3.982 & 19.794 & 3.988 & 19.837 \\
\hline $\mathrm{DyZn}_{3} \mathrm{P}_{3}$ & Hexagonal & $\mathrm{Pb}_{3} / m m c$ & 3.985 & 19.771 & 3.988 & 19.784 \\
\hline
\end{tabular}




\section{Conclusions}

In order to obtain the conditions optimal for synthesizing a new filled skutterudite compound $\mathrm{Tb}_{x} \mathrm{Co}_{4} \mathrm{Sb}_{12}$ and layered rare-earth zinc phosphides $R \mathrm{Zn}_{3} \mathrm{P}_{3}(R=\mathrm{Y}$ and Dy) under high pressures, we have performed in-situ XRD experiments at HTHP. We could obtain the optimum condition for synthesizing the compounds at 2 4 GPa. In this manner, in-situ XRD measurements at HTHP are splendid method for deciding the condition to acquire only target material free from secondary or impurity phases for solid-phase reaction synthesis under high pressure. Furthermore, we developed new integrated-type plastic frame for MA6-6 by using 3D printer. It was confirmed that the MA6-6 system with the plastic frame can be used without problem. This will make it much easier to assemble MA6-6.

\section{Conflict of interest}

There are no conflicts to declare.

\section{Acknowledgements}

This work was carried out using the facilities of the Institute for Solid State Physics, the University of Tokyo. This work has been carried out under the approval of the Photon Factory Program Advisory Committee (Proposal Nos. 2017G164 and 2015G031).

\section{References}

1. H. Sato, H. Sugawara, Y. Aoki, H. Harima, Handbook of Magnetic Materials, North-Holland: Amsterdam, 2009, 18. 1-110.

2. G.S. Nolas, D.T. Morelli, T.M. Tritt, Annu. Rev. Mater. Sci., 1999, 29, 89-116.

3. R.D. Blaugher, J.K. Hulm, J. Phys. Chem. Solids, 1965, 26, $2037-$ 2039.

4. H. Barz, H.C. Ku, G.P. Meisner, Z. Fisk, B.T. Matthias, Proc. Natl. Acad. Sci. U.S.A., 1980, 77, 3132-3134.

5. I. Shirotani, M. Takaya, I. Kaneko, C. Sekine, T. Yagi, Solid State Commun., 2000, 116, 683-686.

6. I. Shirotani, M. Takaya, I. Kaneko, C. Sekine, T. Yagi, Physica C, 2001, 357-360, 329-332.
7. Z. Yu, Y. Wang, Z. Sun, X. Li, A. Wang, D. M. Camaioni, J. Lercher, Green Chem., 2018, 20, 609-619.

8. C. Sekine, Y. Mori, Jpn. J. Appl. Phys., 2017, 56, 05FA09/1-6.

9. C. Sekine, T. Kachi, T. Yoshida, R. Abe, T. Namiki, K. Akahira, K. Ito, J. Phys.: Conf. Ser., 2010, 215, 012141/1-4.

10. C. Sekine, H. Kato, M. Kanazawa, Y. Kawamura, K. Takeda, M. Matsuda, K. Kihou, C.H. Lee, H. Gotou, J. Phys.: Conf. Ser., 2014, 502, 012017/1-4.

11. N. Mandel, J. Donohue, Acta Crystallogr., Sect. B, 1971, 27, 2288-2290.

12. G. J. Snyder, E. S. Toberer, Nat. Mater., 2008, 7, 105-114.

13. G.S. Nolas, J. L. Cohn, G.A. Slack, Phys. Rev. B, 1998, 58, $164-$ 170.

14. J. Yang, Q. Hao, H. Wang, Y. Lan, Q. He, A. Minnich, D. Wang, J. Harriman, V. Varki, M. Dresselhaus, G. Chen, Z. Ren, Phys. Rev. B, 2009, 80, 115329.

15.V.L. Kuznetsov, L.A. Kuznetsova, D.M. Rowe, J. Phys.. Condens. Matter, 2003, 15, 5035-5048.

16. W. Jeitschko, A. J. Foecker, D. Paschke, M. V. Dewalsky, C. B. H. Evers, B. Kunnen, A. Lang, G. Kotzyba, U. C. Rodewald, and M. H. Möller, Z. Anorg. Allg. Chem., 2000, 626, 1112-1120.

17. Z.G. Mei, W. Zhang, L.D. Chen, J. Yang, Phys. Rev. B, 2006, 74, 153202/1-4.

18. A.T. Nientiedt, W. Jeitschko, J. Solid State Chem., 1999, 146, 478-483.

19. T. Kimura, T. Goto, H. Shintani, K. Ishizaka, T. Arima, Y. Tokura, Nature, 2003, 426, 55-58.

20. R. Ramesh, N.A. Spaldin, Nat. Mater, 2007, 6, 21-29.

21. K. Taniguchi, N. Abe, T. Takenobu, Y. Iwasa, T. Arima, Phys. Rev. Lett., 2006, 97, 097203.

22. Y. Yamasaki, S. Miyasaka, Y. Kaneko, J.-P. He, T. Arima, Y. Tokura, Phys. Rev. Lett., 2006, 96, 207204.

23. A. Yamada, N. Hara, K. Matsubayashi, K. Munakata, C. Ganguli, A. Ochiai, T. Matsumoto, Y. Uwatoko, J. Phys. Conf. Ser., 2010, $215,012031$.

24. N. Nishiyama, Y. Wang, T. Sanehira, T. Irifune, M. L. Rivers, High Pressure Res., 2008, 28, 307-314. 